\title{
Assessment of the Effect of Fatty Infiltration on Hepatic FDG Uptake
}

\author{
(D) Tamer Özülker, (D) Filiz Özülker
}

University of Health Sciences, Istanbul Okmeydanı Training and Research Hospital, Department of Nuclear Medicine, istanbul, Turkey

\section{Abstract}

Objective: In fluorodeoxyglucose (FDG) positron emission tomography (PET)/computerized tomography (CT) studies, physiological liver FDG uptake has been used as a reference in the evaluation of FDG uptake in pathological processes, even in malignancies. There is an ongoing debate on the effect of liver attenuation and FDG uptake in the liver. We aimed to evaluate the possible effect of fatty infiltration on the standardized uptake value (SUV) of the liver.

Methods: A total of 88 patients were included in this study. Subjects were divided into 2 groups by calculating the Hounsfield unit (HU) of the liver from the unenhanced CT part of the PET/CT study and comparing it with that of the spleen. Fatty liver group included 42 patients (26 female, 16 male) with a mean age of $59.6 \pm 11.6$ years, while control group consisted of 46 patients ( 22 female, 24 male) with a mean age of $60.2 \pm 11$ years. The patients with a mean liver attenuation value (in $\mathrm{HU}$ ) equal and greater than that of spleen were enrolled in the control group, while the patients with a mean liver attenuation value lower than the spleen were included in the fatty liver group. A subset of patients in the fatty liver group with difference in attenuation between the liver and spleen of more than $10 \mathrm{HU}$ (HUS-HUL > 10) were evaluated separately. Age, weight, history of diabetes mellitus (DM) and chemotherapy, serum alanine aminotransferase (ALT) and aspartate aminotransferase (AST) levels, simultaneous blood glucose levels during PET scan and the elapsed time between FDG injection and the beginning of PET scan were recorded.

Results: The average SUVmean and SUVmax values were $2.7 \pm 0.7$ and $3.6 \pm 0.9$ in the fatty liver group, $2.8 \pm 0.7$ and $3.8 \pm 1$ in the HUS-HUL $>10$ group and $3.3 \pm 0.6$ and $4.4 \pm 0.9$ in the control group, respectively. The average SUVmean and SUVmax values of the fatty liver group and HUS-HUL $>10$ group were significantly different from the control group $(p<0.05)$. The patients in the fatty liver group had higher ALT $(p=0.025)$ and glucose levels $(p=0.001)$, weight $(p=0.001)$, and DM rate $(p=0.002)$ compared to the control group.

Conclusion: Hepatic steatosis causes a statistically significant decrease in SUVmean and SUVmax values in liver. Therefore, we must be careful when using the liver as an internal reference organ

Keywords: Fluorine 18-fluorodeoxyglucose, hepatosteatosis, positron emission tomography, standardized uptake value

\section{INTRODUCTION}

The use of standardized uptake values (SUVs) in determining malignant nature of lesions, aggressiveness of malignancies and therapy response in clinical fluorine $18\left({ }^{18} \mathrm{~F}\right)$-fluorodeoxyglucose (FDG)-positron emission tomography (PET)/computerized tomography (CT) oncology imaging is increasing. SUV is a semiquantitative measurement that corresponds to measured activity normalized for body weight/surface area and injected dose. The formula for calculating SUV is region of interest (ROI) activity (mCi/mL) x body weight (g)/injected dose (mCi). Although the application of SUV eliminates some degree of uncertainty in patient size and the amount of injected FDG, it is still liable to many weaknesses that can cause misleading results. SUV, rather than being an absolute value in characterization of lesions, is a proportional value without units, so to measure the amount of tumoral FDG uptake, there is always a need for a region in 
the body supposed to have normal FDG uptake. The liver has long been used as a reference organ for this purpose (1-4). If the ${ }^{18} \mathrm{~F}-\mathrm{FDG}$ uptake in the target lesion is greater than the liver in terms of SUV, the hypermetabolic focus is considered abnormal.

Fatty liver disease reflects a wide spectrum of conditions characterized histologically by excessive accumulation of triglycerides and cholesterols within the cytoplasm of hepatocytes. Fatty infiltration of the liver is further subdivided as alcoholic fatty liver disease or non-alcoholic fatty liver disease (NAFLD). NAFLD is the most common chronic liver disease in the developed countries with an estimated prevalence of 20\%-30\% in adult populations $(5,6)$. NAFLD includes two pathological entities as simple steatosis and non-alcoholic steatohepatitis (NASH). NASH is a more serious condition that can eventually progress to cirrhosis and promote hepatocellular carcinoma (7).

Several studies have been conducted to investigate the possible effect of fatty infiltration on liver SUVs (8-15). Some of these studies have reported no correlation between low attenuation due to high fat content of liver and ${ }^{18}$ F-FDG uptake $(10,13)$. In one study, FDG PET showed a significant negative correlation between the severity of fatty liver and SUVmax of the liver (15). Increased FDG uptake due to steatohepatitis was also reported in other studies $(9,14,16)$.

These contradictory results have led us to investigate the relation between fatty infiltration of the liver and FDG uptake in terms of SUVmax and SUVmean values.

\section{METHODS}

\section{Patients}

FDG PET/CT examinations performed at our institution between September 2016 and April 2017 were evaluated retrospectively by investigating the patients' medical charts. Due to being retrospective, study approval by the clinical research ethics committee is waived, but the study was approved by the local institutional review board. A total of 88 patients were enrolled in this study. Patients with liver metastasis or previous liver disease that could effect hepatic uptake were not included in this study. Subjects were divided into 2 groups by calculating the Hounsfield unit (HU) of the liver from the unenhanced CT part of the PET/CT study and comparing it with that of the spleen. The fatty liver group included 42 patients ( 26 female, 16 male) with a mean age of $59.6 \pm 11.6$ years. The control group consisted of 46 patients (22 female, 24 male) with a mean age of $60.2 \pm 11$ years. The patients with a mean liver attenuation value (in Hounsfield units) equal and greater than that of spleen were enrolled in the control group, while the patients with a mean liver attenuation value lower than the spleen were included in the fatty liver group. A subset of patients in the fatty liver group with difference in attenuation between the liver and spleen of more than $10 \mathrm{HU}$ (HUS-HUL >10) were evaluated separately. Age, weight, history of diabetes mellitus (DM) and chemotherapy, serum alanine aminotransferase (ALT) and aspartate aminotransferase (AST) levels, simultaneous blood glucose levels during PET scan and the elapsed time between FDG injection and the beginning of PET scan were recorded. Primary malignancies of the patients were lung cancer in 12 patients (13.6\%) colorectal cancer in eight patients (9\%), breast cancer in 24 patients (27.2\%), bladder cancer in six patients (6.8\%), head and neck cancer in six patients (6.8\%), sarcoma in five patients (5.6\%), gynecological malignancies in 14 patients (15.9\%), skin cancer in five patients (5.6\%), neuroendocrine tumor in one patient (1.1\%), cancer of unknown primary in four patients (4.5\%), gastrointestinal stromal tumor in one patient (1.1\%), multiple myeloma in one patient $(1.1 \%)$ and thyroid cancer in one patient (1.1\%).

\section{Imaging}

$\mathrm{PET} / \mathrm{CT}$ images were obtained using an integrated PET/CT scanner consisting of a full-ring HI-REZ LSO PET and a six-slice CT scanner (Siemens Biograph 6, Chicago, IL). Patients were instructed to fast for at least 6 hours before ${ }^{18} \mathrm{~F}-\mathrm{FDG}$ injection. Blood glucose levels were measured before the study and ${ }^{18} \mathrm{~F}$-FDG was injected only when the blood glucose level was below $11.11 \mathrm{mmoL} / \mathrm{L}$. Patients were injected with 296-555 MBq ${ }^{18} \mathrm{~F}$-FDG based on body weight. After waiting 50 minutes relaxed in a semi reclining chair, the patients were visualized using an integrated PET/CT scanner. The CT part of the study was performed without an intravenous contrast medium to define only anatomical landmarks and make attenuation correction on the PET images. The CT scan was performed first with the following parameters: $50 \mathrm{mAs}, 140 \mathrm{kV}$ and 5-mm section thickness. Whole body CT was performed in the craniocaudal direction. The images were obtained with the arms of the patients raised to avoid false increase in liver FDG uptake due to beam-hardening effects.

\section{Measurement of SUV and HU values}

A 2-cm diameter ROI was placed over the right lobe of the liver. Same ROIs were plotted on the PET and CT scans of the liver avoiding any lesions, biliary, vascular structures and artifacts. For each ROI, SUVmean and SUVmax of the liver were measured with the formula "ROI activity $(\mathrm{mCi} / \mathrm{mL}) \mathrm{x}$ body weight/injected dose (mCi)". Mean attenuation value (HUmean) were also measured from the ROI plotted on CT part of the study (Figure 1). 


\section{Statistical Analysis}

IBM SPSS Statistics version 22 (IBM Corp., Armonk, NY, USA) program was used for statistical analysis. The normality of the parameters was evaluated by the Shapiro-Wilk test. Student's t-test or Mann-Whitney U test was used for comparison between two groups, where appropriate. Regarding SUVmean and SUVmax values, comparison between the control group and
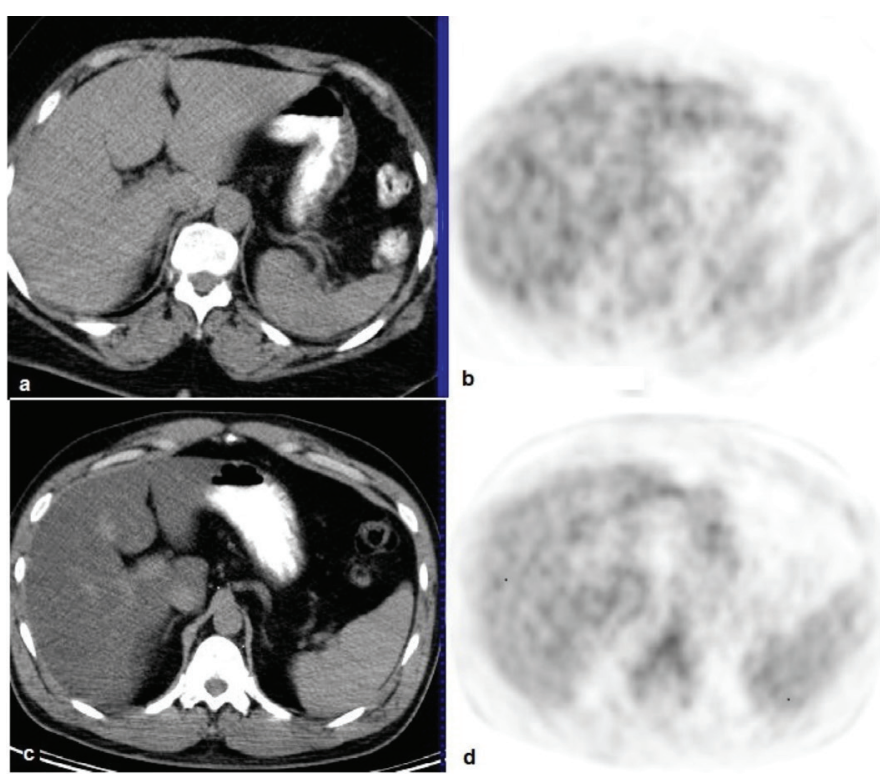

Figure 1. (a, b) Axial computerized tomography and pozitron emisyon tomografi sections of a patient from the control group, (c, d) and fatty liver group the fatty liver disease group was done using Student's t-test. Fisher's exact chi-square test and Continuity (Yates) correction were used for the comparison of qualitative data of gender, DM and chemotherapy status, gamma-glutamyl transferase, AST and ALT elevation, elapsed time and glucose levels. Significance was assessed at $p<0.05$.

\section{RESULTS}

Average liver SUVmax and SUVmean were significantly lower in patients with fatty liver compared to the control group $(p<0.05)$. Average spleen SUVmax and Spleen SUVmean were also significantly lower in patients with fatty liver compared to the control group $(p<0.05)$ (Table 1$)$. In addition, the average liver SUVmax and SUVmean were significantly lower in patients in the subset of fatty liver group (HUS-HUL $>10$ ) compared to the control group $(\boldsymbol{p}<0.05)$ (Table 2). The comparison of data of fatty liver and control groups in terms of clinical parameters are presented in Table 3. The fatty liver group showed a significantly higher mean body weight $(84.95 \pm 13.76 \mathrm{~kg})$ than the control group $(74.45 \pm 14.28 \mathrm{~kg})$. There were 16 patients (38.1\%) with DM in the fatty liver group, while there were four diabetic patients (8.7\%) in the control group. Serum ALT values were significantly higher in the fatty liver group than the control group. Serum glucose levels were also higher in the fatty liver group (115.74 \pm 33.11$)$ than the control group (91.63 \pm 14.4$)$.

\begin{tabular}{|l|l|l|l|}
\hline \multicolumn{2}{|l|}{ Table 1. Evaluation of groups } & \multicolumn{2}{l|}{} \\
\hline & $\begin{array}{l}\text { Patients with fatty liver } \\
(\mathbf{n}=\mathbf{4 2})\end{array}$ & Controls (n=46) & \\
\cline { 2 - 3 } & Mean \pm SD & Mean \pm SD & $0.001^{*}$ \\
\hline Liver SUVmax & $3.61 \pm 0.97$ & $4.41 \pm 0.94$ & $0.001^{*}$ \\
\hline Liver SUVmean & $2.70 \pm 0.70$ & $3.34 \pm 0.66$ & $0.001^{*}$ \\
\hline Liver mean HU & $36.43 \pm 9.63$ & $57.08 \pm 6.36$ & $0.008^{*}$ \\
\hline Spleen SUVmean & $2.29 \pm 0.63$ & $2.62 \pm 0.48$ & $0.028^{*}$ \\
\hline Spleen SUVmax & $2.93 \pm 0.76$ & $3.27 \pm 0.66$ & $0.001^{*}$ \\
\hline Spleen mean HU & $47.29 \pm 5.59$ & $40.46 \pm 9.16$ & \\
\hline Student t-test* $p<0.05$ SD: Standard deviation, Max: Maksimum, SUV: Standardized uptake value, HU: Hounsfield unit & \\
\hline
\end{tabular}

Table 2. Evaluation of groups

\begin{tabular}{|l|l|l|l|}
\hline \multirow{2}{*}{} & $\begin{array}{l}\text { Patients with severe fatty } \\
\text { liver (n=23) }\end{array}$ & Control (n=46) & \multirow{2}{*}{} \\
\cline { 2 - 3 } & Mean \pm SD & Mean \pm SD & \\
\hline Liver SUVmax & $3.84 \pm 1.10$ & $4.41 \pm 0.94$ & $0.028^{*}$ \\
\hline Liver SUVmean & $2.87 \pm 0.79$ & $3.34 \pm 0.66$ & $0.010^{*}$ \\
\hline Liver mean HU & $32.08 \pm 10.39$ & $57.08 \pm 6.36$ & $0.001^{*}$ \\
\hline Student $t$-test* $\mathrm{p}<0.05$ SD: Standard deviation, Max: Maksimum, SUV: Standardized uptake value, HU: Hounsfield unit & \multicolumn{2}{|l}{} \\
\hline
\end{tabular}




\section{DISCUSSION}

Non-invasive methods that distinguish benign lesions from malignancies have always been searched. FDG PET has been utilized for this purpose depending on the fact that malignant lesions generally have a higher glucose consumption rate and thus higher FDG uptake. Initially, semiquantitative SUV measurements, as an indicator of amount of FDG uptake, seemed to be a robust method in the characterization of malignant lesions and regarded by some authors as "metabolic biopsy" $(17,18)$. However, in clinical practice, there have been numerous limitations, such as partial volume and spillover effects, attenuation correction, reconstruction method and parameters for scanner type, count noise bias effect, elapsed time between radiotracer injection and imaging, competing transport effects and body size (19). Therefore, it is not realistic to rely on a certain static SUVmax threshold to distinguish benign lesions from malignant ones. The qualitative visual interpretation of 18F-FDG uptake by using liver as a reference standard became a common practice to overcome this shortcoming. Fatty liver disease, which means accumulation of fat in the form of triglycerides and cholesterol in the liver cells, theoretically might cause a decrease in the uptake of FDG in hepatocytes. This potential decline in FDG has significant clinical implications as a result of misinterpretation of FDG-positive lesions, as it shows itself as a reduction in SUVmax compared to normal livers, therefore, we tried to assess whether liver ${ }^{18} \mathrm{FDG}$ uptake was affected by hepatosteatosis. In our study, we found that average liver SUVmax and SUVmean of patients with fatty liver were significantly lower than the control group $(p<0.05)$. In addition, average liver SUVmax and SUVmean in the subset of fatty liver group (HUS-HUL $>10$ ) were significantly lower than the control group $(p<0.05)$. In the literature, conflicting results have been reported by several studies investigating the relationship between hepatic steatosis and hepatic FDG uptake. One of the oldest studies conducted for this purpose by Qazi et al. (20) reported that liver SUVmax/spleen SUVmax ratio of the fatty liver group was significantly lower than that of the control group (1.1 vs $1.4, p=0.002$ ). There were limitations for this preliminary report, such as the relatively small number of subjects enrolled in the study and measurement of SUVmax instead of SUVmean which may give rise to less reliable results in the evaluation of a large organ like liver. In their prospective case-control study, Abikhzer et al. (11) analyzed the effect of fatty infiltration on hepatic metabolic activity in 37 patients. The authors found that patients with hepatic steatosis had significantly lower hepatic metabolic activity in terms of SUVmax measurements compared with control subjects, when the SUV is corrected for lean body mass and not for body weight. Even though the results were statistically significant, the degree of the change in SUVmax values was not found satisfactory by authors to be accepted as clinically significant.

Lin et al. (15) reported that hepatic steatosis had a significant negative effect on hepatic metabolic activity as measured by SUVmax. They retrospectively analyzed ${ }^{18} \mathrm{~F}$-FDG PET studies of 173 patients who were investigated for non-oncological diseases. They divided the patients into four groups according to the ultrasonography findings: no fatty liver, mild, moderate and severe. The mean SUVmax of liver in subjects with no, mild, moderate

\begin{tabular}{|c|c|c|c|}
\hline & $\begin{array}{l}\text { Patients with fatty liver } \\
(n=42)\end{array}$ & Controls $(n=46)$ & $\mathrm{p}$ \\
\hline Age: mean \pm SD & $59.69 \pm 11.61$ & $60.22 \pm 11.04$ & ${ }^{1} 0.828$ \\
\hline \multicolumn{4}{|l|}{ Gender, n (\%) } \\
\hline Female & $26(61.9 \%)$ & $22(47.8 \%)$ & \multirow[t]{2}{*}{${ }^{2} 0.267$} \\
\hline Male & 16 (38.1\%) & 24 (52.2\%) & \\
\hline Diabetes mellitus, n (\%) & $16(38.1 \%)$ & $4(8.7 \%)$ & ${ }^{2} 0.002^{*}$ \\
\hline ALT elevation, $n$ (\%) & $7(16.7 \%)$ & $1(2.2 \%)$ & ${ }^{3} 0.025^{*}$ \\
\hline AST elevation, $n(\%)$ & $2(4.8 \%)$ & $0(0 \%)$ & ${ }^{3} 0.225$ \\
\hline GGT, n (\%) & $7(16.7 \%)$ & $5(10.9 \%)$ & ${ }^{2} 0.631$ \\
\hline Chemotherapy, n (\%) & $12(28.6 \%)$ & $13(28.3 \%)$ & 21.000 \\
\hline Elapsed time: mean \pm SD & $70.48 \pm 15.94$ (65) & $76.72 \pm 17.35(70)$ & ${ }^{4} 0.049 *$ \\
\hline Glucose: mean \pm SD & $115.74 \pm 33.11(109)$ & $91.63 \pm 14.40(89.5)$ & ${ }^{4} 0.001^{*}$ \\
\hline Weight: mean \pm SD & $84.95 \pm 13.76$ & $74.45 \pm 14.28$ & ${ }^{1} 0.001^{*}$ \\
\hline
\end{tabular}


and severe fatty liver were $3.13 \pm 0.49,3.08 \pm 0.45,3.01 \pm 0.44$, and $2.43 \pm 0.27$, respectively. Differences in SUVmax were statistically significant. They concluded that the liver could not be used as a comparator of increased FDG activity in the lesions of patients with fatty liver disease. These findings are in accordance with our results, indicating a negative relationship between SUVmax and $\mathrm{HU}$ values. However, there are also other reports in the literature that contradict our findings. Pak et al. (21) retrospectively analyzed FDG PET/CT studies of 96 consecutive patients who were screened for cancer and found no significant difference in liver SUVmean and SUVmax between controls and fatty liver group.

Dostbil et al. (13) assessed the relationship between fatty infiltration of liver and hepatic metabolic activity in 79 patients with hepatosteatosis on ${ }^{18} \mathrm{FDG} \mathrm{PET} / \mathrm{CT}$. The control group in the study included 77 patients with a mean liver HU value greater than mean spleen $\mathrm{HU}$ value and the patient group included 79 patients in whom the mean liver HUvalue was lower than or equal to the mean spleen $\mathrm{HU}$ value. The authors further divided the patient group into subsets according to their degree of hepatic steatosis. There was no statistically significant difference between the mean and maximum liver SUVs in patients with fatty liver disease and the control group. Abele and Fung (10) conducted a study to evaluate the association between diffuse fatty infiltration and average FDG uptake, with the assumption that hepatocyte expansion due to fat accumulation may lead to a decrease in SUVmean. The average SUVmean for the control group was $2.18 \pm 0.36$ and this value was not significantly different for the groups of fatty liver disease $(2.03 \pm 36)$ and more strictly defined subset of fatty liver disease $(2.07 \pm 0.24)$ groups.

Some authors described a controversial increase in liver SUVmean values in patients with fatty liver. Liu et al. (12) reported a positive relationship between liver SUVmean and fatty infiltration when the severity was mild to moderate, while there is a negative effect when more severe. They also noted that FDG uptake of liver gradually increase in patients as the body mass index (BMI) increases from underweight to overweight, but a decrease in SUVmean values occur when the patient is obese.

High levels of ${ }^{18} \mathrm{~F}$-FDG uptake in inflammatory cells are well known, and this has led to use of FDG PET as a potential imaging modality in infectious diseases. Keramida et al. (14) reported that FDG uptake in the liver is increased in NASH due to irreversible uptake in inflammatory cells superimposed on reversible hepatocyte uptake. Bural et al. (22) compared hepatic SUVs and hepatic metabolic volumetric products (HMVP) among patients with diffuse hepatic steatosis and control subjects with normal liver. They found an increase in HMVP as a result of increased hepatic metabolic activity likely related to the inflammatory process in diffuse hepatic steatosis. Increased FDG uptake in the liver with high fat content could be accounted for the increased activity of Kupffer cells, a kind of macrophage that acts by engulfing FDG (23). This accumulation of FDG uptake at focal hepatic steatosis can cause a diagnostic dilemma in imaging by mimicking metastasis $(24,25)$. Conversely, focal fat spared area in a liver with diffuse fatty infiltration can demonstrate focal FDG uptake masquerading as liver metastases, probably when steatosis is not accompanied with inflammation $(26,27)$

In our study, we found a statistically significant difference between the body weight $(p<0.001)$, serum ALT levels $(0.025)$, DM status $(0.002)$, and glucose levels $(p<0.001)$ of the patients with fatty liver and the control group. There may be a positive correlation between serum liver enzyme levels and SUVs of liver on FDG PET that can affect diagnostic sensitivity of hepatic malignant or infectious lesions on FDG PET (28). Patients with fatty liver disease have higher AST and ALT levels (21). Although patients in the fatty liver group in our study had higher serum enzyme levels, we could not detect any positive relation between SUVs and ALT and AST levels. It is known that BMI levels are higher in patients with fatty liver than in normal patients (13, 21). In our study, we could not calculate BMI of patients since we did not get their height values, but mean body weight of the patients with hepatic steatosis were significantly higher. All of the subjects in the patient and control groups had oncological diseases and there was not any statistically significant difference between the two groups in terms of chemotherapy history. Lin et al. (8) found that age had a significant and positive effect on both the maximum and mean SUVs of the liver in FDG PET imaging. In our study, the mean ages of the patients were not significantly different between the two groups.

Interestingly, average spleen SUVmean and SUVmax of patients with fatty liver were also significantly lower than the control group $(p<0.05)$ in our study. This issue needs to be clarified by additional studies.

We preferred to rely on unenhanced CT part of the PET CT in the diagnosis of fatty liver, as assessment of liver attenuation by use of unenhanced $\mathrm{CT}$ represents an objective and non-invasive mean for detection of asymptomatic hepatic steatosis $(29,30)$. The diagnosis could be done with biopsy and histopathology and this can be a limitation of our study.

\section{CONCLUSION}

Contrary to most studies reported in the literature, hepatic steatosis causes a statistically significant decrease in SUVmean and SUVmax values in liver, unless it is associated with 
inflammatory conditions as in NASH. Therefore, we must be careful while using the liver as an internal reference organ.

\section{Ethics}

Ethics Committee Approval: Due to being retrospective, study approval by the clinical research ethics committee is waived, but the study was approved by the local institutional review board.

Informed Consent: Retrospective study.

Peer-review: Externally peer-reviewed.

\section{Author Contributions}

Surgical and Medical Practices: T.Ö., F.Ö., Concept: T.Ö., F.Ö., Design: T.Ö., F.Ö., Data Collection or Processing: T.Ö., F.Ö., Analysis or Interpretation: T.Ö., F.Ö., Literature Search: T.Ö., Writing: T.Ö., F.Ö.

Conflict of Interest: The authors have no conflicts of interest to declared.

Financial Disclosure: The authors declared that this study has received no financial support.

\section{REFERENCES}

1. KhandaniAH, Wahl RL. Applications of PET in liver imaging. Radiol Clin North Am 2005;43:849-60.

2. Kumar R , Xiu Y, Yu JQ, Takalkar A, El-Haddad G, Potenta S et al . 18 F-FDG PET in evaluation of adrenal lesions inpatients with lung cancer. J Nucl Med 2004; $45: 2058-62$

3. van KouwenMC, Jansen JB, van Goor H, de Castro S, Oyen WJ, Drenth JP. FDG-PETis able to detect pancreatic carcinoma in chronic pancreatitis. Eur J Nucl Med Mol Imaging 2005;32:399-404

4. Barrington SF, Mikhaeel NG, Meignan M, Hutchings M, Müeller SP,Kostakoglu $\mathrm{L}$, et al. Role of imaging in thestaging and response assessment of lymphoma: consensus of theinternational conference on malignant lymphomas imaging working group. J Clin Oncol 2014;32:3048-58.

5. Wah-Kheong C, Khean-Lee G. Epidemiology of a fast emerging disease in the Asia-Pacific region: non-alcoholic fatty liver disease. Hepatol Int 2013;7:65-71.

6. Papamiltiadous ES, Roberts SK, Nicoll AJ, Ryan MC, Itsiopoulos C, Salim A, et al. A randomised controlled trial of a Mediterranean Dietary Intervention for Adults with Non Alcoholic Fatty Liver Disease (MEDINA): study protocol. BMC Gastroenterol 2016;16:14.

7. European Association for the Study of the Liver (EASL); European Association for the Study of Diabetes (EASD); European Association for the Study of Obesity (EASO) EASL-EASD-EASO Clinical Practice Guidelines for the management of non-alcoholic fatty liver disease. J Hepatol 2016;64:1388402.

8. Lin CY, Ding HJ, Lin CC, Chen CC, Sun SS, Kao CH. Impact of age on FDG uptake in the liver on PET scan Clin Imaging 2010;34:348-50.

9. Keramida G, Potts J, Bush J,Dizdarevic S, Peters AM. Hepatic steatosis is associated with increased hepatic FDG uptake. Eur J Radiol 2014;83:751-5.

10. Abele JT, Fung Cl. Effect of hepatic steatosis on liver FDG uptake measured in mean standard uptake values. Radiology 2010;254:917-24.

11. Abikhzer G, Alabed YZ, Azoulay L, Assayag J, Rush C. Altered hepatic metabolic activity in patients with hepatic steatosis on FDG PET/CT. AJR Am J Roentgenol 2011;196:176-80.
12. Liu G, Li Y, Hu P, Cheng D, Shi H. The combined effects of serum lipids, BMI, and fatty liver on 18F-FDG uptake in the liver in a large population from China: an 18F-FDG-PET/CT study. Nucl Med Commun 2015;36:709-16.

13. Dostbil Z, Varoğlu E, Serdengeçti M, Kaya B, Onder H, Sari O. Evaluation of hepatic metabolic activity in non-alcoholic fatty livers on 18FDG PET/CT.Rev Esp Med Nucl Imagen Mol 2013;32:156-61.

14. Keramida G, Potts J, Bush J, Verma S, Dizdarevic S, Peters AM. Accumulation of (18)F-FDG in the liver in hepatic steatosis.AJR Am J Roentgenol 2014;203:643-8. Erratum in: AJR Am J Roentgenol 2015;204:1137.

15. Lin CY, Lin WY, Lin CC, Shih CM, Jeng LB, Kao CH. The negative impact of fatty liver on maximum standard uptake value of liver on FDG PET. Clin Imaging 2011;35:437-41.

16. Kim YH, Kim JY, Jang SJ, Chung HW, Jang KS, Paik SS, et al. F-18 FDG uptake in focal fatty infiltration of liver mimicking hepatic malignancy on PET/CT images. Clin Nucl Med 2011;36:1146-8.

17. Hain SF, Curran KM, Beggs AD, Fogelman I, O'Doherty MJ, Maisey MN. FDGPET as a "metabolic biopsy" tool in thoracic lesions with indeterminate biopsy. Eur J Nucl Med 2001;28:1336-40.

18. Beggs AD, Hain SF, Curran KM, O'Doherty MJ. FDG-PET as a "metabolic biopsy" tool in non-lung lesions with indeterminate biopsy. Eur J Nucl Med Mol Imaging 2002;29:542-6.

19. Kwee TC, Cheng G, Lam MG, Basu S, Alavi A. SUVmax of 2.5 should not be embraced as a magic threshold for separating benign from malignant lesions. Eur J Nucl Med Mol Imaging 2013;40:1475-7.

20. Qazi F, Oliver D, Nguyen N, Osman M. Fatty liver: impact on metabolic activity as detected with18F FDG-PET/CT. J Nucl Med 2008;49:263.

21. Pak K, Kim SJ, Kim IJ, Kim K, Kim H, Kim SJ. Hepatic FDG Uptake is not Associated with Hepatic Steatosis but with Visceral Fat Volume in Cancer Screening. Nucl Med Mol Imaging 2012;46:176-81.

22. Bural GG, Torigian DA, Burke A, Houseni M, Alkhawaldeh K, Cucchiara A, et al. Quantitative assessment of the hepatic metabolic volume product in patients with diffuse hepatic steatosis and normal controls through use of FDG-PET and MR imaging: a novel concept. Mol Imaging Biol 2010;12:233-9.

23. Kim YH, Kim JY, Jang SJ, Chung HW, Jang KS, Paik SS, et al. F-18 FDG uptake in focal fatty infiltration of liver mimicking hepatic malignancy on PET/CT images. Clin Nucl Med 2011;36:1146-8.

24. Le Y, Chen Y, Huang Z,Cai L, Zhang L. Intense FDG activity in focal hepatic steatosis. Clin Nucl Med 2014;39:669-72.

25. Han N, Feng $\mathrm{H}$, Arnous MM, Bouhari A, Lan X. Multiple liver focal fat sparing lesions with unexpectedly increased (18)F-FDG uptake mimicking metastases examined by ultrasound (18)F-FDG PET/CT and MRI. Hell J Nucl Med 2016;19:173-5.

26. Harisankar CN. Focal fat sparing of the liver: a nonmalignant cause of focal FDG uptake on FDG PET/CT. Clin Nucl Med 2014;39:359-61.

27. Purandare NC, Rangarajan V, Rajnish A, Shah S, Arora A, Pathak S. Focal fat spared area in the livermasquerading as hepatic metastasis on F-18 FDG PET imaging. Clin Nucl Med 2008;33:802-5.

28. Lin CY, Ding HJ, Lin T, Lin CC, Kuo TH, Kao CH. Positive correlation between serum liver enzyme levels and standard uptake values of liver on FDG-PET. Clin Imaging 2010;34:109-12.

29. Boyce CJ, Pickhardt PJ, Kim DH, Taylor AJ, Winter TC, Bruce RJ, et al. Hepatic steatosis (fatty liver disease) in asymptomatic adults identified by unenhanced low-dose CT. AJR Am J Roentgeno I2010;194:623-8.

30. Kodama Y, Ng CS, Wu TT, Ayers GD, Curley SA, Abdalla EK, et al. Comparison of CT methods for determining the fat content of the liver. AJR Am J Roentgenol 2007; $188: 1307-12$ 